\title{
Fluctuations of Conserved Quantities
}

\author{
Volker Koch* \\ Lawrence Berkeley National Laboratory \\ Berkeley, CA, 94707, USA \\ E-mail: vkoch@lbl.gov
}

We will discuss fluctuations and correlations of conserved quantities in the context of heavy ion collisions and high temperature QCD. We will argue that these quantities are useful to study the properties of high temperature QCD matter such as its quasiparticple content relevant degrees of freedem etc. Also question such as equilibration in heavy ion collisions can be addressed.

The 2nd edition of the International Workshop - Correlations and Fluctuations in Relativistic Nuclear Collisions -

July 7-9 2006

Galileo Galilei Institute, Florence, Italy

* Speaker. 


\section{Introduction}

The study and analysis of fluctuations and correlations are an essential method to characterize a physical system. In general, one can distinguish between several classes of fluctuations. On the most fundamental level there are quantum fluctuations, which arise if the specific observable does not commute with the Hamiltonian of the system under consideration. These fluctuations probably play less a role for the physics of heavy ion collisions. Second, there are "dynamical" fluctuations reflecting the dynamics and responses of the system. They help to characterize the properties of the bulk (semi-classical) description of the system. Examples are density fluctuations, which are controlled by the compressibility of the system. Finally, there are "trivial" fluctuations induced by the measurement process itself, such as finite number statistics etc. These need to be understood, controlled and subtracted in order to access the dynamical fluctuations which tell as about the properties of the system.

Fluctuations are also closely related to phase transitions. The well known phenomenon of critical opalescence is a result of fluctuations at all length scales due to a second order phase transition. First order transitions, on the other hand, give rise to bubble formation, i.e. density fluctuations at the extreme.

The most efficient way to address fluctuations of a system created in a heavy ion collision is via the study of event-by-event (E-by-E) fluctuations, where a given observable is measured on an event-by-event basis and the fluctuations are studied over the ensemble of the events. In most cases (namely when the fluctuations are Gaussian) this analysis is equivalent to the measurement of two particle correlations over the same region of acceptance [1]. Consequently, fluctuations tell us about the 2-point functions of the system, which in turn determine the response of the system to external perturbations.

\section{Fluctuations of conserved charges}

In the framework of statistical physics, which appears to describe the bulk properties of heavy ion collisions up to RHIC energies, fluctuations measure the susceptibilities of the system. These susceptibilities also determine the response of the system to external forces. For example, by measuring fluctuations of the net electric charge in a given rapidity interval, one obtains information on how this (sub)system would respond to applying an external (static) electric field. In other words, by measuring fluctuations one gains access to the same fundamental properties of the system as "table top" experiments dealing with macroscopic probes. In the later case, of course, fluctuation measurements would be impossible.

In a thermal system, fluctuations (variances) and correlations (co-variances) are given by the second derivatives of the free energy, the so called susceptibilities. Given a partition function

$$
Z=\operatorname{tr}\left(\exp \left(-\beta\left(H-\sum_{i} \mu_{i} Q_{i}\right)\right)\right)
$$

and free energy $F=-T \log (Z)$ of a system with conserved charges $Q_{i}$ and associated chemical potentials $\mu_{i}$ the susceptibilities are given by

$$
\chi_{i, j}=-\frac{1}{V} \frac{d F}{d \mu_{i} \mu_{j}} .
$$


They are related to the (co)-variance via

$$
\left\langle\delta Q_{i} \delta Q_{j}\right\rangle=T V \chi_{i, j}
$$

Examples discussed in the context of heavy ion collisions are net charge fluctuations [2, 3]

$$
\left\langle(\delta Q)^{2}\right\rangle=T V \chi_{Q, Q}=-\frac{d F}{d \mu_{Q} \mu_{Q}}
$$

and Baryon-Strangeness or Charge-Strangeness correlations [ [4, 5]

$$
\begin{aligned}
\langle\delta B \delta S\rangle & =T V \chi_{B, S} \\
\langle\delta Q \delta S\rangle & =T V \chi_{Q, S}
\end{aligned}
$$

just to name a few examples. For a thermal system these susceptibilities can be determined directly by Lattice QCD (LQCG) methods (see e.g. [6, 7]). In reference [6] even higher derivatives of the free energy with respect to the quark chemical potentials have been determined which provide further insight into the system and its degrees of freedom. These fluctuation are well defined observables, but some of them for example those involving Baryon number are not easily measured as they would require the detection of e.g. neutrons. In addition, special care needs to be taken in order to correct for global charge conservation [8, 97. Charge fluctuations make only sense in a grand-canonical description, which can be realized in experiment if only a small subsystem is being considered. This may lead to some limitations in detecting interesting fluctuations. For example in the case of net charge fluctuations, it has been predicted that a QGP should lead to reduced net charge fluctuations per entropy [月, 的. If, for simplicity one characterizes the size of the system to be analyzed in experiment by its range in rapidity $\Delta Y_{\text {accept }}$, then one has the following conditions for which such a measurement is meaningful

$$
\begin{gathered}
\Delta Y_{\text {accept }} \ll \Delta Y_{\text {total }} \\
\Delta Y_{\text {kick }} \ll \Delta Y_{\text {accept }}
\end{gathered}
$$

where $\Delta Y_{\text {total }}$ is the total width of the charge particle distribution and $\Delta Y_{k i c k}$ denotes the shift in rapidity a charge particle receives during the evolution of the system, i.e. hadronization and subsequent decay of hadronic resonances. If the conditions (2.6) are met, corrections due to global charge conservation are small $\left(\Delta Y_{\text {accept }} \ll \Delta Y_{\text {total }}\right)$. In addition, since $\Delta Y_{\text {kick }} \ll \Delta Y_{\text {accept }}$, in a given event the evolution of the system does not change the charge of the subsystem and, therefore, the eventby event ensemble does reflect the ensemble of a QGP, if produced in these collisions. In other words, the fluctuations become frozen in. If, on the other hand, $\Delta Y_{\text {kick }} \simeq \Delta Y_{\text {accept }}$, charges are transported in and out of the acceptance range and thus the observed fluctuations are those of the final (hadronic state). If, as is the case at SPS-energies, $\Delta Y_{\text {total }} \simeq \Delta Y_{\text {kick }} \simeq \Delta Y_{\text {accept }}$, the measured charge fluctuations cannot be easily compared with those obtained in LQCD and other models based on the grand-canonical ensemble [9, 10]. This might be the explanation, why at SPS-energies the net charge fluctuations do barely show the correlations due to resonances [11, 12] and why at RHIC only a resonance gas is observed [13, 14]. These considerations become especially important if one wants to search for the QCD critical point, which is expected to be located at the lowest SPS 
energies. In this case the conditions (2.6) are certainly not fulfilled and different methods to extract possible critical fluctuations of the baryon number need to be developed.

Aside from these experimental subtleties, the study of fluctuations or susceptibilities provide also theoretical insight into the matter at high temperature. As an example lets consider the recently proposed correlation between baryon number and strangeness [4]

$$
C_{B S} \equiv-3 \frac{\sigma_{B S}}{\sigma_{S}^{2}}=-3 \frac{\langle B S\rangle-\langle B\rangle\langle S\rangle}{\left\langle S^{2}\right\rangle-\langle S\rangle^{2}}=-3 \frac{\langle B S\rangle}{\left\langle S^{2}\right\rangle} .
$$

which is unity for a simple QGP, or more generally, for a system where the quark flavors are uncorrelated. For a hadron gas on the other hand,

$$
C_{B S} \approx 3 \frac{\langle\Lambda\rangle+\langle\bar{\Lambda}\rangle+\ldots+3\left\langle\Omega^{-}\right\rangle+3\left\langle\bar{\Omega}^{+}\right\rangle}{\left\langle K^{0}\right\rangle+\left\langle\bar{K}^{0}\right\rangle+\ldots+9\left\langle\Omega^{-}\right\rangle+9\left\langle\bar{\Omega}^{+}\right\rangle},
$$

and the numerator receives contributions from only (strange) baryons, while the denominator receives contributions also from (strange) mesons. As a result, $C_{B S}=0.66$ for $T=170 \mathrm{MeV}$ and $\mu_{B}=0$. On the other hand, at very high $\mu_{B}$ and low $T$, where strangeness is carried exclusively by Lambdas and Kaons, $C_{B S} \approx \frac{3}{2}$. This significant dependence of $C_{B S}$ on the hadronic environment is in sharp contrast to the simple quark-gluon plasma where the correlation coefficient remains strictly unity at all temperatures and chemical potentials.

The correlation coefficient $C_{B S}$ can be expressed in terms of basic quark-flavor susceptibilities

$$
\chi_{f f^{\prime}}=-\frac{1}{V} \frac{\partial^{2} F}{\partial \mu_{f} \partial \mu_{f}^{\prime}}, C_{B S}=1+\frac{\chi_{d s}+\chi_{u s}}{\chi_{s s}}
$$

and thus is calculable in lattice QCD. As is evident from the above expression, the deviation of $C_{B S}$ from unity is controlled by the ratio of flavor-off-diagonal over flavor-diagonal susceptibilities. Or in other words, this ratio measures the strength of flavor correlations. For the specific case of light-strange susceptibilities, the values $\chi_{f f^{\prime}}$ extracted at $T=1.5 T_{c}$ by Gavai et al. [15], we obtain $\left(\chi_{u s}+\chi_{d s}\right) / \chi_{s s}=0.00(3) / 0.53(1) \ll 1$, and thus $C_{B S} \approx 1$, suggesting that the quark flavors are uncorrelated. Unfortunately, at present no results at lower temperatures for $2+1$ flavor QCD are available. But since the relevant quantity is the ratio of flavor-off-diagonal over flavordiagonal susceptibilities, similar arguments can be made in the light-quark sector only, where the necessary susceptibilities have been calculated over a wide range of temperature [6]. The relevant ratio $-2 \chi_{u d} / \chi_{u u}$ is shown in Fig. 1. While the ratio is finite below $T_{c}$, reflecting the presence of correlations in a hadron gas, it drops rapidly around $T_{c}$ and is consistent with zero above $T \geq 1.1 T_{c}$ indicating that the up and down flavors are uncorrelated above this temperature.

Recently there has appeared an interesting model [16] that describes the chromodynamic system as a gas of massive quarks, anti-quarks, and gluons together with a myriad of their bound states generated by a screened Coulomb potential. In order to assess the consistency of this model with present lattice results, we estimate the ratio $C_{B S}$ in such a scenario. Using the masses and degeneracies of the various states as given in Ref. [16] we find $C_{B S} \approx 0.62$ at $T=1.5 T_{c}$, which is significantly below the value of $C_{B S} \approx 1$ obtained from lattice QCD. Essential for the deviation is the presence of $q \bar{q}$-bound states in the model, which, like the mesons in the hadron gas, contribute to the denominator but not the numerator of $C_{B S}$. Note, that if there were as many di-quark states 


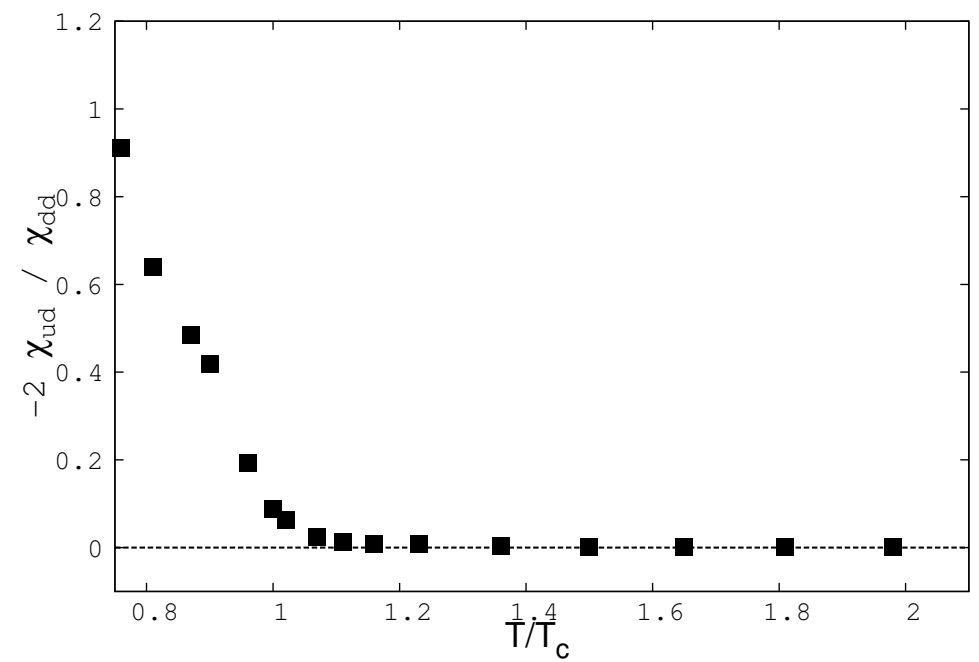

Figure 1: Ratio of off-diagonal to diagonal susceptibility as a function of temperature in 2-flavor unquenched Lattice QCD

as quark-antiquark states, then $C_{B S}=1$. However, this is unlikely due to the structure of the colorcoulomb interaction, which favors $\bar{q}-q$ states. Of course the presence of baryonic states above $T_{C}$ would change the picture somewhat. However, there are additional susceptibilities extracted from LQCD and a careful analysis of those will provide useful information about the structure and the relevant degrees of freedom of hot QCD matter.

\section{Equilibrium}

Finally a few remarks regarding equilibrium in heavy ion collisions. The success of the hadron gas model [17] suggests that equilibrium is a good assumption at least for the final state. In addition, measurements of fluctuations seem to agree with the hadron gas model. However, it is not yet clear from the data over which volume equilibrium is reached. Absent any correlations the partition functions factorizes, and one cannot distinguish between a system made out of small equilibrated subsystems Fig. 2(a) and one where a large part is equilibrated Fig. 2(b)

The necessary correlations required in order to differentiate between these two possibilities are for example provided by conserved quantum numbers. For instance strangeness is always produced locally, and thus it takes some time for it to equilibrate over a large volume. This has been realized by Redlich et al. [18]. They considered a canonical ensemble and introduced a strangeness correlation volume, defined as the volume over which strangeness is conserved. They found that for small strangeness correlation volumina, $V_{\text {corr }}<20 V_{\text {nucleon }}$ the strangeness abundance does not scale with overall volume size, whereas for large correlations volumina it scales. In other words for $V_{\text {corr }} \gg 20 V_{\text {nucleon }}$ the grand-canonical description is a reasonable one. The relevant experimental data are shown in Fig. 3 where the enhancement per participant of strange baryons and anti-baryons is shown as a function of the number of participant nucleons, $N_{\text {part }}$, i.e centrality. For both SPS and RIHC energies, the strangeness enhancement keeps increasing with centrality up to the higest values of $N_{\text {part }}$ ! Assuming $N_{\text {part }}$ is a measure for the volume of the system, a fully 


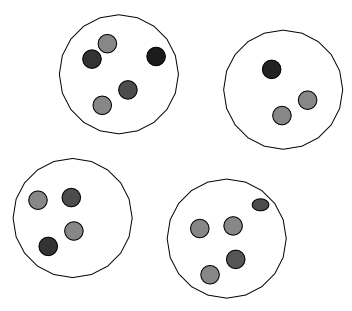

(a)

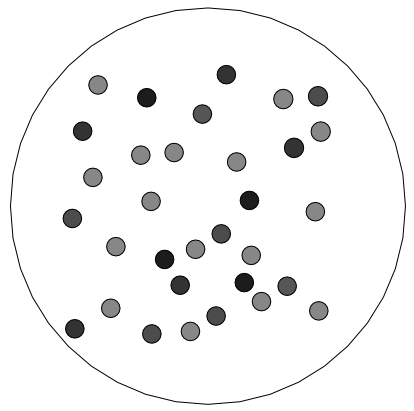

(b)

Figure 2: Individual nucleon-nucleon collisions (a) and "true" matter generated in a nucleus-nucleus collision (b).

equilibrated system would lead to a strangeness enhancement per participant which is independent of $N_{\text {part }}$, contrary to what is seen in the data. Comparing with findings of [18], where an increase of similiar fashion is seen for correlation volumina $V_{\text {corr }}<20 V_{\text {nucleon }}$ one is lead to the conclusion that equilibrium has not been established over regions larger than $V \simeq 20 V_{\text {nucleon }}$ even for the highest centralities. Again fluctuations and correlations can be utilized to investigate this question further.
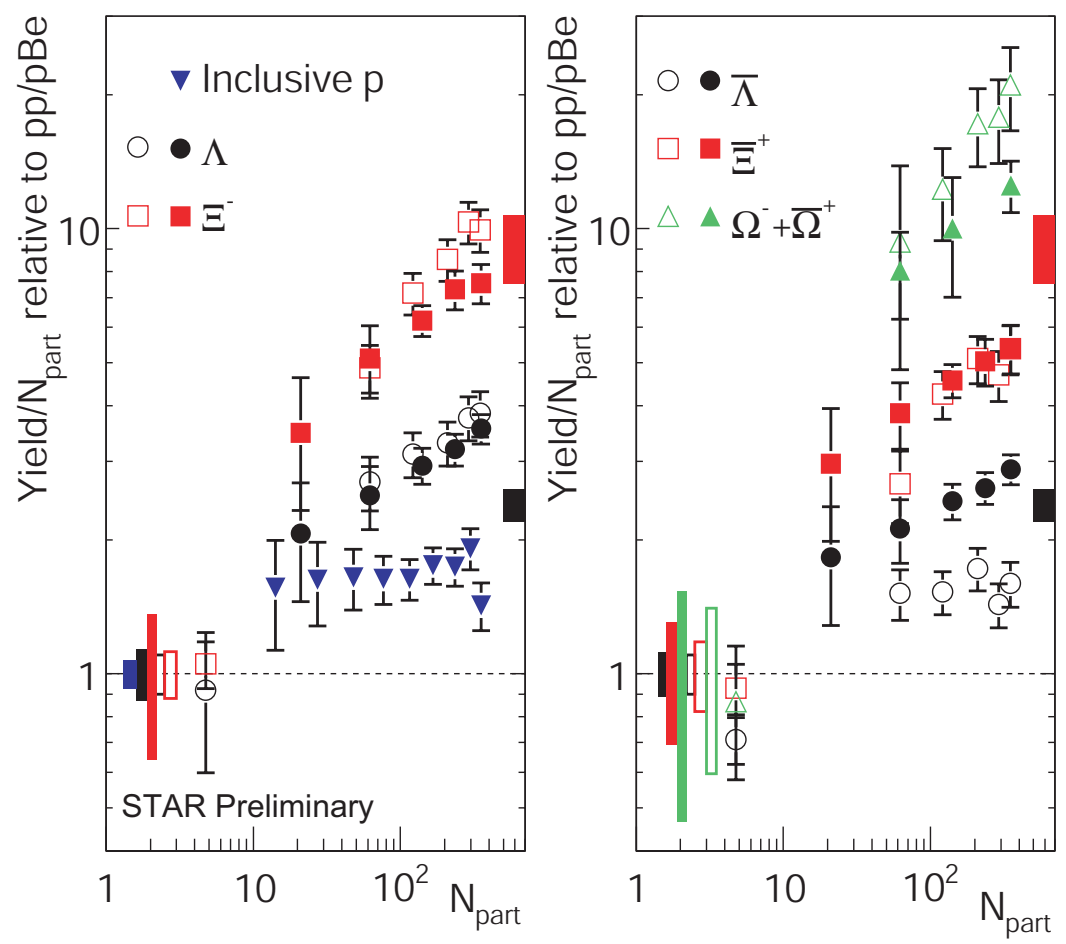

Figure 3: Strangeness enhancement as function of number of participants for strange baryons (left) and anti-baryons (right). Solid symbols are for Au-Au collisions at $\sqrt{s}=200 \mathrm{GeV}$ [19], and the open symbols are measured by NA57 from $\mathrm{Pb}-\mathrm{Pb}$ at $\sqrt{s}=17.3 \mathrm{GeV}$ [20]. Figure adapted from [19].

As demonstrated in [21] a definitive measurement of equilibration at RHIC energies would require 
the measurement of five Omega-baryon coincidences. To which extend this is feasible remains to be seen. Two particle correlations, which are often discussed as a possible means to establish the degree of equilibrium, are dominated by Poisson statistics and thus are misleading.

\section{Summary}

In this contribution we have discussed the physics of fluctuations in the context of heavy ion collisions. As this is a developing field, this should be considered as a snapshot of our present understanding. We have argued that fluctuations are indeed a useful tool to investigate the properties of the matter created in these collisions. As an example we have shown that baryon-strangeness correlations can tell us about the underlying degrees of freedom and their correlations of the high temperature phase. We have also pointed out that a meaningful measurement of fluctuations of conserved quantities requires that a small subsystem is considered, which in turn should be large compared to correlation introduced by the dynamics of the collision. Finally we discussed the issue of equilibration and pointed out that the observed rise in strangeness enhancement per participant is not really consistent with a fully equilibrated system. Rather it seems to indicate that strangeness is equilibrated over volumina smaller than $20 V_{\text {nucleon }}$. Further progress in this area can be made by investigating fluctuations, however, at RHIC energies, it would require rather difficult measurements of many (>5) particle correlations.

\section{Acknowledgments}

This work was supported by the Director, Office of Science, Office of High Energy and Nuclear Physics, Division of Nuclear Physics, and by the Office of Basic Energy Sciences, Division of Nuclear Sciences, of the U.S. Department of Energy under Contract No. DE-AC03-76SF00098.

\section{References}

[1] A. Bialas and V. Koch, Event by event fluctuations and inclusive distributions, Phys. Lett. B456 (1999) 1-4, [http://arXiv.org/abs/nucl-th/9902063].

[2] S. Jeon and V. Koch, Charged particle ratio fluctuation as a signal for qgp, Phys. Rev. Lett. 85 (2000) 2076-2079, http://arXiv.org/abs/hep-ph/0003168].

[3] M. Asakawa, U. W. Heinz, and B. Muller, Fluctuation probes of quark deconfinement, Phys. Rev. Lett. 85 (2000) 2072-2075, hhttp://arXiv.org/abs/hep-ph/0003169].

[4] V. Koch, A. Majumder, and J. Randrup, Baryon-strangeness correlations: A diagnostic of strongly interacting matter, Phys. Rev. Lett. 95 (2005) 182301, hucl-th/0505052].

[5] R. V. Gavai and S. Gupta, Fluctuations, strangeness and quasi-quarks in heavy-ion collisions from lattice qcd, Phys. Rev. D73 (2006) 014004, hep-lat/ 0510044 ].

[6] C. R. Allton et al., Thermodynamics of two flavor qcd to sixth order in quark chemical potential, Phys. Rev. D71 (2005) 054508, [hep-lat/0501030].

[7] R. V. Gavai and S. Gupta, Quark number susceptibilities, strangeness and dynamical confinement, Phys. Rev. D64 (2001) 074506, [hep-lat/0103013]. 
[8] M. Bleicher, S. Jeon, and V. Koch, Event-by-event fluctuations of the charged particle ratio from non-equilibrium transport theory, Phys. Rev. C62 (2000) 061902, [http://arXiv.org/abs/hep-ph/0006201].

[9] S.-y. Jeon and V. Koch, Event-by-event fluctuations, hep-ph/0304012.

[10] J. Zaranek, Measures of charge fluctuations in nuclear collisions, Phys. Rev. C66 (2002) 024905, [hep-ph/0111228].

[11] CERES Collaboration, H. Appelshauser et al., Event-by-event fluctuations at sps, Nucl. Phys. $\mathbf{A 7 5 2}$ (2005) 394-397, [nucl-ex/0409022].

[12] NA49 Collaboration, C. Alt et al., Electric charge fluctuations in central pb + pb collisions at 20-agev, 30-agev, 40-agev, 80-agev and 158-agev, Phys. Rev. C70 (2004) 064903, [nucl-ex/0406013].

[13] PHENIX Collaboration, K. Adcox et al., Net charge fluctuations in au + au interactions at $s(n n) * *(1 / 2)=130-$ gev, Phys. Rev. Lett. 89 (2002) 082301, [http://arXiv.org/abs/nucl-ex/0203014].

[14] STAR Collaboration, J. Adams et al., Multiplicity fluctuations in au + au collisions at $s(n n) * *(1 / 2)=$ 130-gev, Phys. Rev. C68 (2003) 044905, nucl-ex/0307007.

[15] R. V. Gavai and S. Gupta, Valence quarks in the qcd plasma: Quark number susceptibilities and screening, Phys. Rev. D67 (2003) 034501, hep-lat/0211015.

[16] E. V. Shuryak and I. Zahed, Towards a theory of binary bound states in the quark gluon plasma, Phys. Rev. D70 (2004) 054507, hep-ph/ 0403127 ].

[17] P. Braun-Munzinger, K. Redlich, and J. Stachel, Particle production in heavy ion collisions, nucl-th/0304013.

[18] S. Hamieh, K. Redlich, and A. Tounsi, Canonical description of strangeness enhancement from $p$ a to pb pb collisions, Phys. Lett. B486 (2000) 61-66, [hep-ph/0006024].

[19] H. Caines, Using strange hadrons as dense matter probes, nucl-ex/0608008.

[20] F. Antinori et al., First results on strange baryon production from the na57 experiment, Nucl. Phys. A698 (2002) 118-126.

[21] A. Majumder and V. Koch, The chemical equilibration volume: Measuring the degree of thermalization, nucl-th/0305047. 